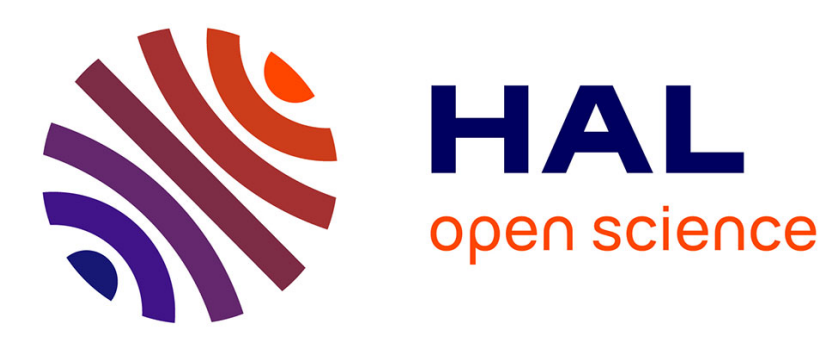

\title{
Structure MESFET AsGa bigrille distribuée. Performances potentielles en amplification
}

\author{
C. Byl, Y. Crosnier, G. Salmer
}

\section{To cite this version:}

C. Byl, Y. Crosnier, G. Salmer. Structure MESFET AsGa bigrille distribuée. Performances potentielles en amplification. Revue de Physique Appliquée, 1988, 23 (7), pp.1221-1228. 10.1051/rphysap:019880023070122100 . jpa-00245934

HAL Id: jpa-00245934

https://hal.science/jpa-00245934

Submitted on 1 Jan 1988

HAL is a multi-disciplinary open access archive for the deposit and dissemination of scientific research documents, whether they are published or not. The documents may come from teaching and research institutions in France or abroad, or from public or private research centers.
L'archive ouverte pluridisciplinaire HAL, est destinée au dépôt et à la diffusion de documents scientifiques de niveau recherche, publiés ou non, émanant des établissements d'enseignement et de recherche français ou étrangers, des laboratoires publics ou privés. 


\title{
Structure MESFET AsGa bigrille distribuée. Performances potentielles en amplification
}

\author{
C. Byl, Y. Crosnier et G. Salmer \\ Centre Hyperfréquences et Semiconducteurs, U.A. 287 C.N.R.S., Bât. P4, Université des Sciences et \\ Techniques de Lille Flandres Artois, 59655 Villeneuve d'Ascq Cedex, France
}

(Reçu le 16 octobre 1987, révisé le 27 novembre 1987, accepté le 8 décembre 1987)

\begin{abstract}
Résumé. - Nous présentons une étude des potentialités en amplification d'une structure MESFET AsGa bigrille distribuée, avec entrée sur la grille $G_{1}$, sortie sur la grille $G_{2}$, le drain étant découplé à la masse. Une modélisation, utilisant le logiciel Spice, permet d'examiner, successivement, les influences des impédances terminales, du développement, des pertes et de la polarisation des lignes de grilles. Une comparaison avec la structure monogrille distribuée est donnée. Enfin, la faisabilité du dispositif amplificateur est établie par des essais pratiqués sur une première réalisation.
\end{abstract}

\begin{abstract}
We present a study on the amplification capabilities of a GaAs distributed dual-gate MESFET structure, with the gate $G_{1}$ as input and the gate $G_{2}$ as output, the drain electrode being R.F. grounded. A model, based on the Spice program, allows us to investigate, successively, the influences of the termination impedances, the width, the losses and the bias conditions of the gate lines. A comparison with the single gate distributed structure is reported. Lastly, the device feasability is demonstrated through measurements carried out from a first realization.
\end{abstract}

\section{Introduction.}

La transposition du principe des amplificateurs distribués directement aux structures MESFETs AsGa à grand développement de grille fait l'objet, depuis plusieurs années, d'études approfondies dans divers laboratoires $[1,2,3,4,5,6]$. L'objectif poursuivi est d'établir si ces structures pourraient présenter des propriétés aussi intéressantes que celles des amplificateurs distribués classiques à composants discrets : gain et puissance appréciables sur une large bande de fréquences, et impédances d'entrée et de sortie de valeurs raisonnables. La structure monogrille a déjà été largement étudiée, en particulier par Hartnagel et al. Il a été démontré qu'elle pouvait effectivement fonctionner en amplification à condition de rendre exploitables les deux modes de propagation en présence par un choix judicieux des impédances terminales et en minimisant les pertes.
La structure bigrille a, par contre, été beaucoup moins étudiée. A notre connaissance, les rares travaux effectués à son sujet l'ont été par Baudrand et al., et ont porté essentiellement sur les applications potentielles comme coupleurs, isolateurs et déphaseurs dans les circuits intégrés monolithiques.

Ayant acquis antérieurement, au Laboratoire, une certaine expérience d'une part sur les MESFETs bigrilles [7] et, d'autre part, sur les amplificateurs distribués [8], il nous a paru intéressant de mettre à profit cette double expérience pour aborder une approche de la structure bigrille distribuée et de ses possibilités d'amplification. Pour effectuer une étude théorique la plus large possible, nous avons choisi une analyse de type circuit plutôt qu'une analyse de type onde et, pour cela, nous avons élaboré une modélisation reposant sur l'utilisation du logiciel Spice. Parallèlement, sur le plan expérimental, nous avons conçu une structure de test permettant, dans un premier temps, de faire une étude de faisabilité. 
2. Amplificateur large bande à bigrille localisé, configuré avec entrée sur la grille $G_{1}$ et sortie sur la grille $\mathbf{G}_{2}$.

La faisabilité d'un amplificateur large bande à partir d'un bigrille classique localisé, avec entrée sur la grille $G_{1}$ et sortie sur la grille $G_{2}$ a été démontrée par Wyrvinski [7]. La figure 1 en montre le dispositif d'étude et un exemple de courbe de gain obtenu avec un composant réalisé par le L.E.P., ayant pour dimensions de ses grilles $G_{1}$ et $G_{2}: 0,7 \mu \mathrm{m} \times$ $300 \mu \mathrm{m}$. Dans cette configuration, le bigrille est normalement polarisé sur les électrodes des deux grilles et du drain mais celui-ci, du point de vue hyperfréquence, est découplé à la masse par une capacité de forte valeur, appelée $C_{\mathrm{D}}$ sur la figure. L'amplificateur, ainsi réalisé, présente un comportement passe-bande, avec environ $12 \mathrm{~dB}$ de gain et $4 \mathrm{GHz}$ de bande passante autour d'une fréquence centrale entre 10 et $15 \mathrm{GHz}$, accordable par la polarisation de la grille $\mathrm{G}_{2}$. Le principe amplificateur de cette configuration du bigrille étant ainsi établi dans le cas d'un composant localisé, nous allons voir, dans ce qui suit, dans quelle mesure il est possible d'exploiter cette configuration dans le cas, cette fois,
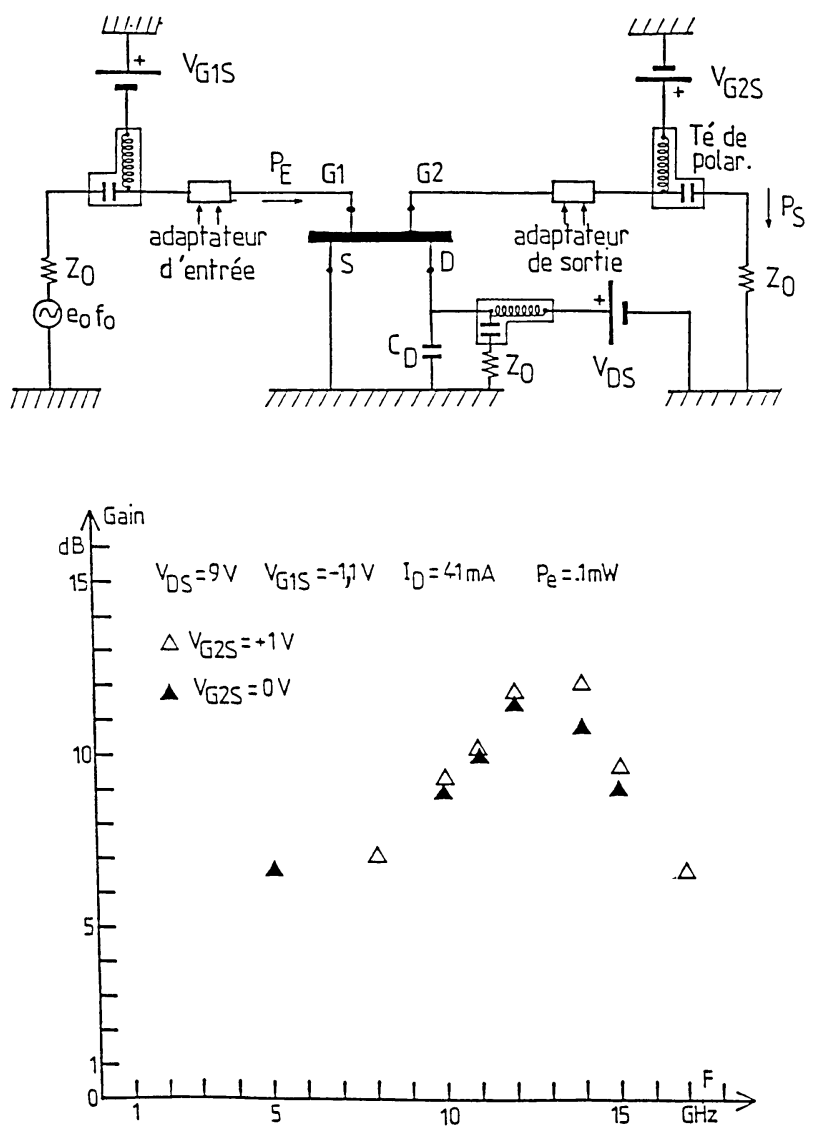

Fig. 1. - Amplificateur à MESFET bigrille localisé en configuration : entrée sur la grille 1 , sortie sur la grille 2 .

[Discrete dual-gate MESFET amplifier with gate 1 as input and gate 2 as output.] d'une structure à grand développement de grille, en mettant à profit les phénomènes de propagation par une utilisation judicieuse des deux lignes constituées par les grilles $G_{1}$ et $G_{2}$.

\section{Dispositif de principe du bigrille distribué et modélisation.}

Le dispositif de principe est représenté sur la figure 2. La source est uniformément à la masse. Le drain est découplé à la masse sur toute sa longueur. Les extrémités 2 et 4 des grilles sont fermées sur des

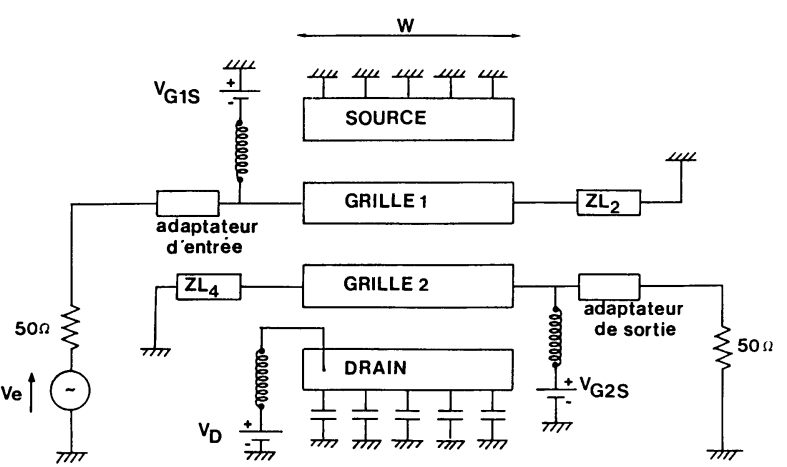

Fig. 2. - Schéma de principe du dispositif amplificateur constitué à partir de la structure bigrille distribuée.

[Principle scheme of the amplifier set-up based on the dual-gate distributed structure.]

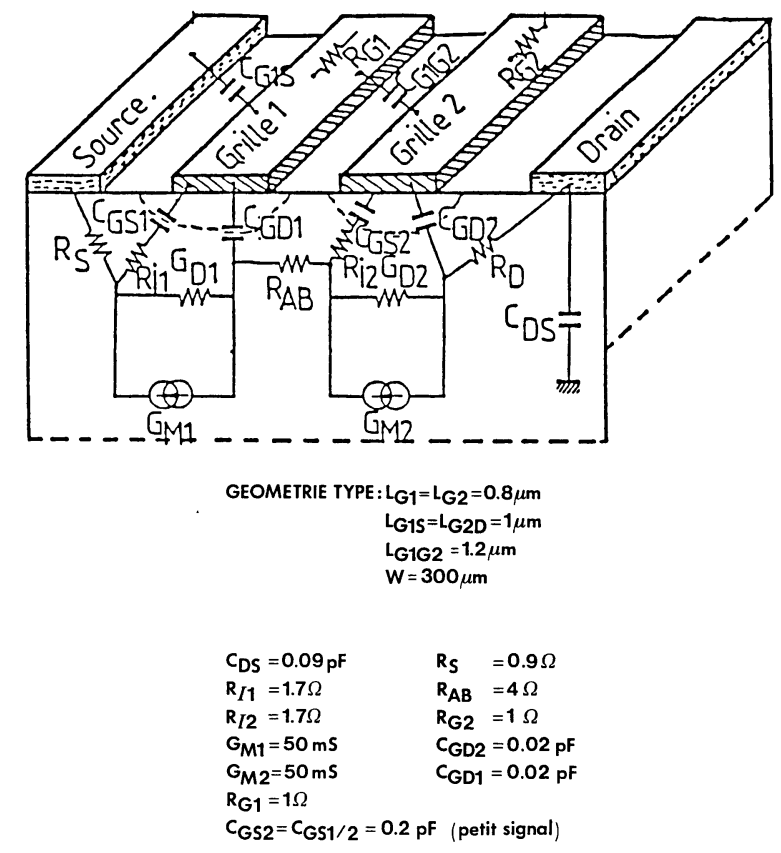

Fig. 3. - Schéma équivalent intrinsèque du MESFET bigrille. (Valeurs des éléments dans le cas d'un L.E.P. BDA01.)

[Intrinsic equivalent circuit of the dual-gate MESFET (the element values are for the L.E.P. BDA01).] 
impédances terminales $Z_{\mathrm{L} 2}$ et $Z_{\mathrm{L} 4}$, tandis que les extrémités 1 et 3 constituent l'entrée et la sortie de la structure et sont connectées, respectivement, à la source et à la charge extérieures (d'impédances $50 \Omega$ ) par l'intermédiaire de transformateurs d'impédance permettant de réaliser l'adaptation.

La modélisation de la structure distribuée a été effectuée en considérant celle-ci comme une cascade de cellules élémentaires, chacune de ces cellules présentant un développement suffisamment petit devant le développement total pour qu'il soit possible de la considérer comme un bigrille localisé. Ce développement de cellule élémentaire a été fixé à $100 \mu \mathrm{m}$ et le schéma qui a été retenu pour représenter le bigrille localisé correspondant est celui, de type cascode, à deux monogrilles, généralement considéré comme étant le mieux représentatif de la réalité physique intrinsèque du composant. Ce schéma équivalent, représenté par la figure 3, comporte un grand nombre d'éléments. Pour ne pas compliquer inutilement la modélisation nous n'avons retenu que les principaux de ces éléments, soit, pour chacun des deux monogrilles : la transconductance $g_{\mathrm{m} i}$, la conductance de sortie $g_{\mathrm{d} i}$, la capacité grille source $C_{\mathrm{gsi}}$ et la capacité grille-drain $C_{\mathrm{gd} i}$. La figure 4 montre, dans ces conditions, comment se présente l'ensemble de la modélisation, incluant les lignes de propagation équivalentes aux deux grilles $\mathrm{G}_{1}$ et $\mathrm{G}_{2}$ sous forme d'éléments selfiques et résistifs $L_{\mathrm{G} i}$ et $R_{\mathrm{G} i}$ répartis et aussi d'une mutuelle inductance répartie $M_{i}=k L_{\mathrm{G} i}$ (les capacités réparties de ces lignes étant supposées comprises dans les éléments $C_{\mathrm{gs} 1}$ et $C_{\mathrm{gd} 2}$ de chaque bigrille élémentaire). Nous avons pris pour valeurs linéiques de ces différents éléments :

$$
\begin{aligned}
\boldsymbol{g}_{\mathrm{m} i} & =150 \mathrm{mS} / \mathrm{mm} & g_{\mathrm{d} i} & =13 \mathrm{mS} / \mathrm{mm} \\
C_{\mathrm{gs} i} & =1 \mathrm{pF} / \mathrm{mm} & C_{\mathrm{gd} i} & =0,08 \mathrm{pF} / \mathrm{mm} \\
L_{\mathrm{G} i} & =0,2 \text { à } 1 \mathrm{nH} / \mathrm{mm} & R_{\mathrm{G} i} & =5 \text { à } 40 \Omega / \mathrm{mm} \\
k & \simeq 0,05 & &
\end{aligned}
$$

ces valeurs étant typiquement celles correspondant à des longueurs des grilles $G_{1}$ et $G_{2}$ de l'ordre de $1 \mu \mathrm{m}$. L'évaluation des paramètres des lignes $L_{\mathrm{G} i}$, $R_{\mathrm{G} i}$ et $k$ est très ardue. En ce qui concerne $R_{\mathrm{G} i}$, nous avons retenu les ordres de grandeurs

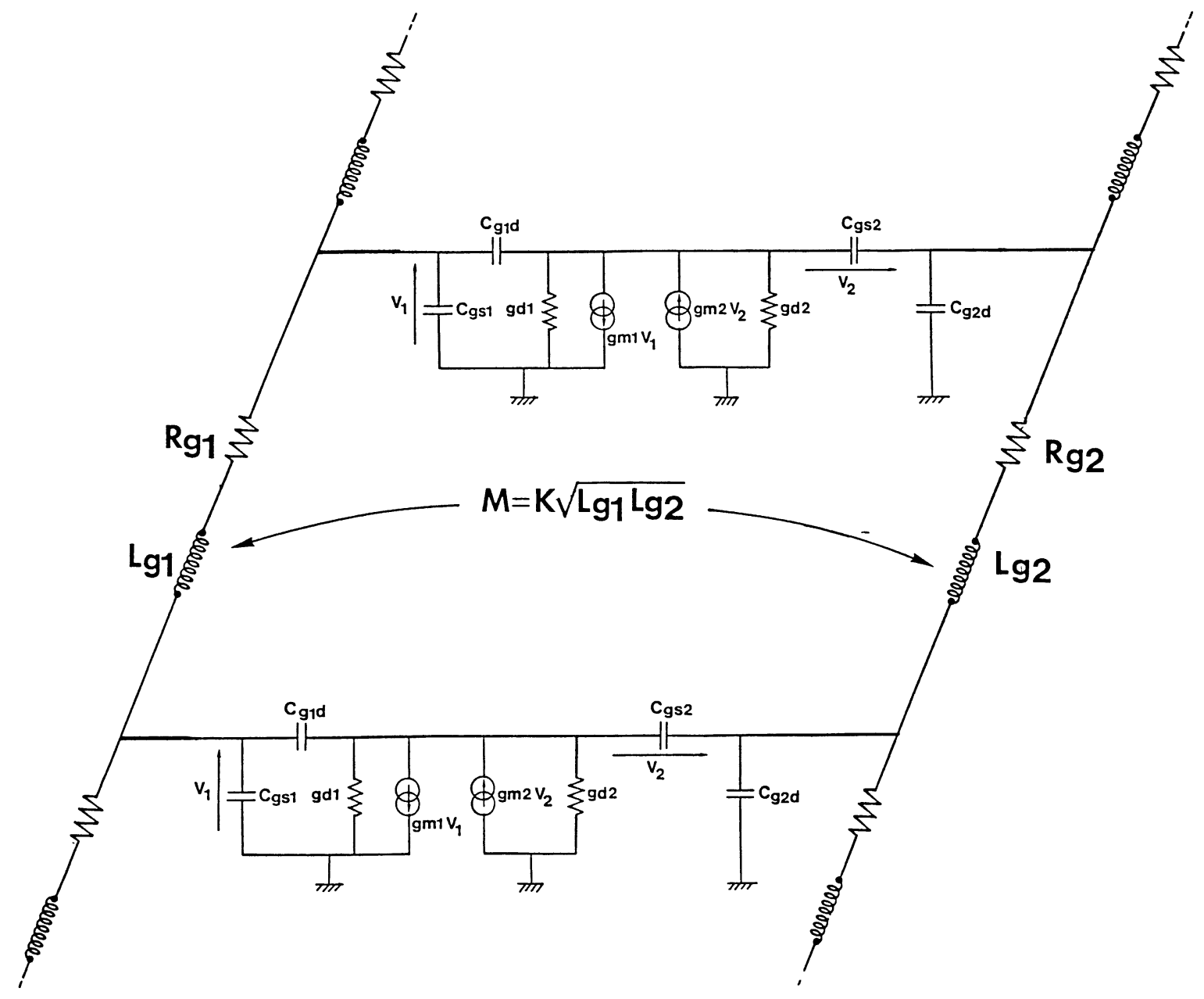

Fig. 4. - Modèle utilisé pour la simulation de la structure bigrille distribuée.

[Model used for the simulation of the dual-gate distributed structure.] 
usuellement obtenus par des mesures en continu. En ce qui concerne $L_{\mathrm{G} i}$ nous avons essentiellement utilisé les indications fournies par la littérature pour des lignes M.I.S. coplanaires [9]. Il est clair qu'il ne peut s'agir que d'une première approximation étant donné que la structure bigrille, avec drain découplé à la masse, s'apparente plutôt à deux lignes couplées coplanaires. Une étude rigoureuse, basée sur les équations de l'électromagnétisme, est actuellement en cours au Laboratoire et devrait permettre de préciser ce point. Enfin, en ce qui concerne le coefficient de couplage magnétique $k$, ne disposant pas, là aussi, d'indications précisément adaptées au cas de notre structure, nous en avons fait une estimation en nous basant sur le cas des lignes microruban couplées pour lequel existe un formalisme de calcul largement répandu [10].

L'ensemble de la structure étant ainsi modélisé, nous avons effectué la simulation de son fonctionnement en régime sinusoïdal petit signal avec le logiciel Spice. Nous avons, ainsi, déterminé les paramètres $S$ en quadripole (entrée en 1 , sortie en $3, Z_{\mathrm{L} 2}$ et $Z_{\mathrm{L} 4}$ étant incluses dans l'ensemble de la structure), le gain maximum en puissance disponible et les impédances d'entrée et de sortie correspondantes. Un traitement ultérieur du fonctionnement de la structure, avec des transformateurs d'impédance, nous a permis de simuler les conditions vraiment représentatives du dispositif réel amplificateur et d'analyser finement, à partir des courants et tensions, le comportement interne en tout point du circuit.

\section{Résultats des simulations.}

4.1 CHOIX DES IMPÉDANCES DE FERMETURE $Z_{\mathrm{L} 2}$ ET $Z_{\mathrm{L} 4}$. - Des nombreux essais effectués, il ressort que l'obtention d'un gain conséquent requiert des impédances de fermeture $Z_{\mathrm{L} 2}$ et $Z_{\mathrm{L} 4}$ dont les coefficients de réflexion (par rapport à $50 \Omega$ ) ont un module voisin de 1 . De plus, pour les développements $W$, de grille, de 1 et $2 \mathrm{~mm}$, que nous avons le plus étudiés, il apparaît que les meilleurs résultats sont généralement obtenus lorsque ces impédances sont selfiques. Une condition similaire a été mise en évidence par Fricke et Hartnagel [11] et Kretschmer et al. [4] dans le cas de la structure monogrille. L'introduction d'une partie résistive dans $Z_{\mathrm{L} 2}$ et $Z_{\mathrm{L} 4}$ conduit systématiquement le dispositif à atténuer au lieu d'amplifier. Et il est tout à fait sans intérêt de prendre des impédances $Z_{\mathrm{L} 2}$ et $Z_{\mathrm{L} 4}$ correspondant aux impédances caractéristiques des lignes des grilles 1 et 2 considérées isolément (l'autre ligne étant supposée à un potentiel nul). Les figures $5 \mathrm{a}$ et $5 \mathrm{~b}$ illustrent ces propriétés avec quelques exemples typiques pour un développement de grille de $1 \mathrm{~mm}$. Il est donc clair que les conditions de fermeture, ainsi mises en évidence, sont très différentes de celles que l'on

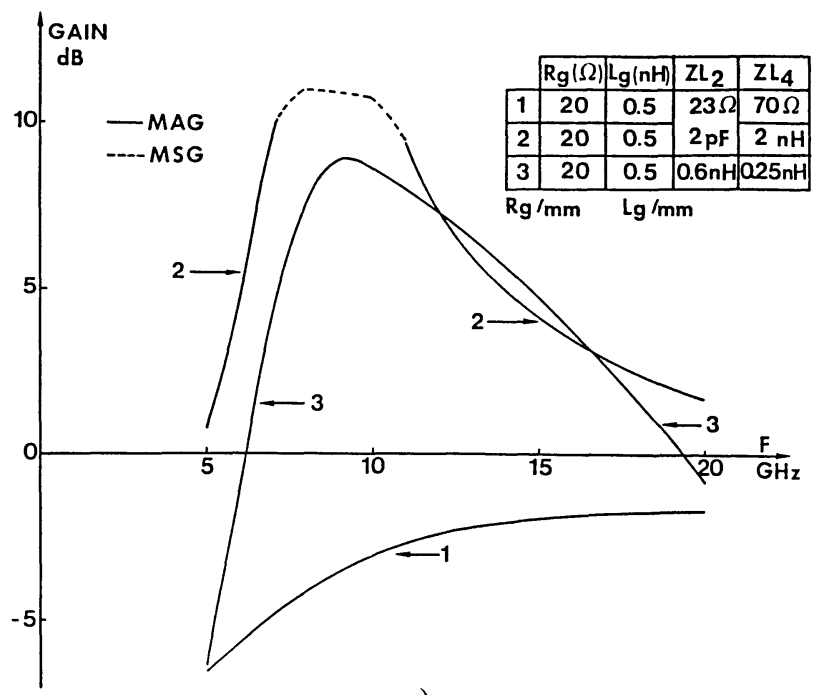

a)

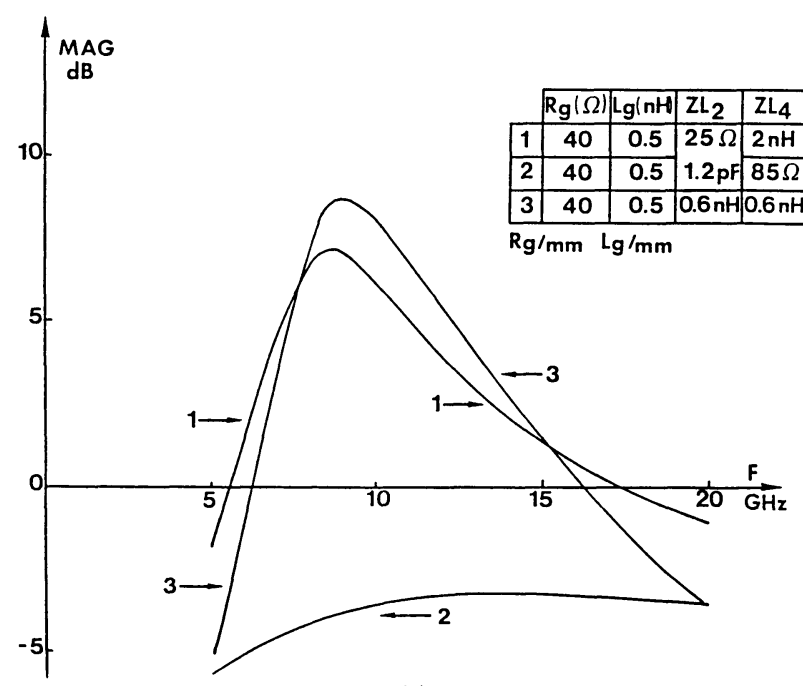

b)

Fig. 5. - Influence des impédances terminales des grilles $G_{1}$ et $G_{2}$ sur le gain de la structure bigrille distribuée pour deux cas de pertes séries des lignes de grille: a) $R_{\mathrm{g}}=20 \Omega$; b) $R_{\mathrm{g}}=40 \Omega$.

[Influence of the gate $G_{1}$ and $G_{2}$ terminal impedances on the gain of the dual-gate distributed structure for two cases of series line losses : a) $R_{\mathrm{g}}=20 \Omega$; b) $R_{\mathrm{g}}=40 \Omega$.]

utilise dans les amplificateurs distribués classiques à composants discrets.

\subsection{INFLUENCE DES PARAMÈTRES DES LIGNES DES} GRILlES $G_{1}$ ET $G_{2}$. - Une étude de sensibilité du gain par rapport aux valeurs des self-inductances et résistances séries $L_{\mathrm{G}}$ et $R_{\mathrm{G}}$ des lignes de grille $\mathrm{G}_{1}$ et $\mathrm{G}_{2}$ nous est apparue intéressante, en raison des variations auxquelles peuvent être assujetties ces grandeurs en fonction de la technologie. Les nombreux essais pratiqués ont tous montré que l'augmentation des inductances ou l'augmentation des résistances séries conduit à un déplacement de la réponse 
de la structure vers les basses fréquences, le gain étant de plus en plus vite limité du côté des hautes fréquences. Des exemples typiques de ces comportements sont donnés sur les figures 6 et 7 pour un développement de $1 \mathrm{~mm}$. En ce qui concerne la première, les impédances $Z_{\mathrm{L} 2}$ et $Z_{\mathrm{L} 4}$ ont été réajustées dans chaque cas pour essayer de conserver une valeur constante du gain maximum. Par contre, en ce qui concerne la seconde, $Z_{\mathrm{L} 2}$ et $Z_{\mathrm{L} 4}$ ont été maintenues constantes.

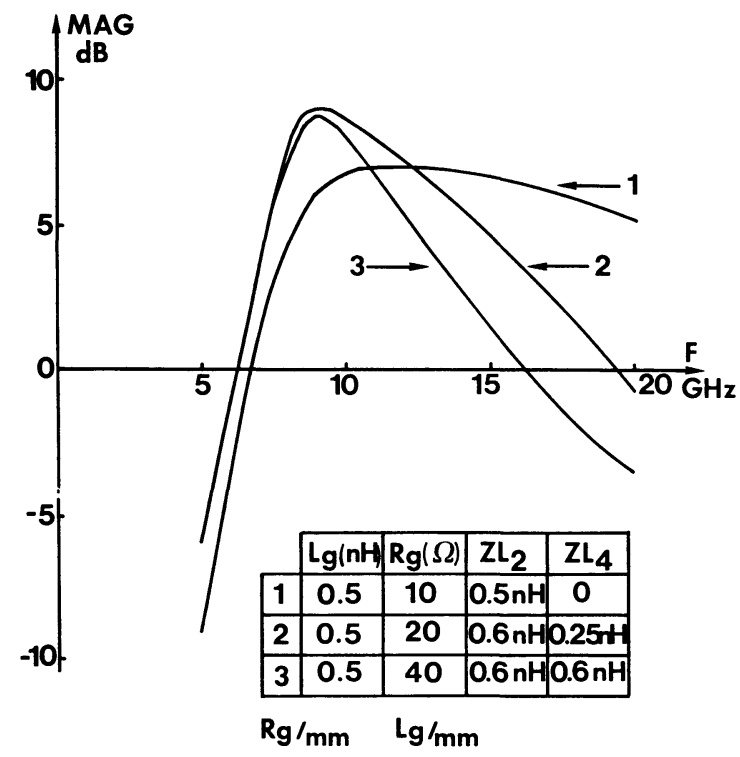

Fig. 6. - Influence des pertes séries des lignes $G_{1}$ et $\mathrm{G}_{2}$ sur le gain de la structure bigrille distribuée (à gain maximum maintenu constant).

[Influence of the series losses of the lines $G_{1}$ and $\mathrm{G}_{2}$ on the gain of the dual-gate distributed structure (with maximum gain kept constant).]

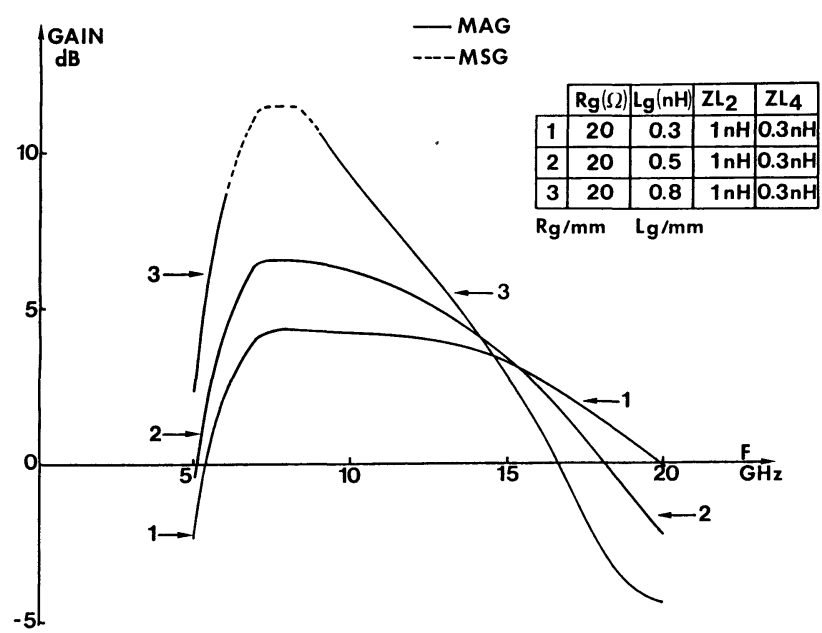

Fig. 7. - Influence des inductances des grilles $G_{1}$ et $\mathrm{G}_{2}$ sur le gain de la structure bigrille distribuée.

[Influence of the inductances of the gate $G_{1}$ and $G_{2}$ on the gain of the dual-gate distributed structure.]
4.3 INFLUENCE DU DÉVELOPPEMENT DE GRILLE $W$. L'augmentation de $W$ est indispensable si l'on souhaite obtenir de la puissance. Une large investigation du comportement du gain en fonction de $W$ a donc été effectuée. Elle a abouti à la conclusion générale que l'augmentation de $W$ a pour effet de déplacer vers les fréquences plus basses la réponse de la structure. C'est ce que montre la figure 8 , pour $W=1,2,3$ et $4 \mathrm{~mm}$. Il semble que, pour les valeurs de $R_{\mathrm{G}}$ et $L_{\mathrm{G}}$ retenues dans ces exemples, un développement $W$ de $2 \mathrm{~mm}$ soit relativement optimal à l'égard de la bande passante. Mais les évolutions du gain montrées sur la figure 8 ne doivent pas être considérées comme nécessairement les meilleures que l'on puisse obtenir en raison de l'extrême sensibilité de la forme de la réponse en fréquence en fonction des impédances de fermeture $Z_{\mathrm{L} 2}$ et $Z_{\mathrm{L} 4}$ et d'une interdépendance étroite entre leur choix et la valeur de $W$.

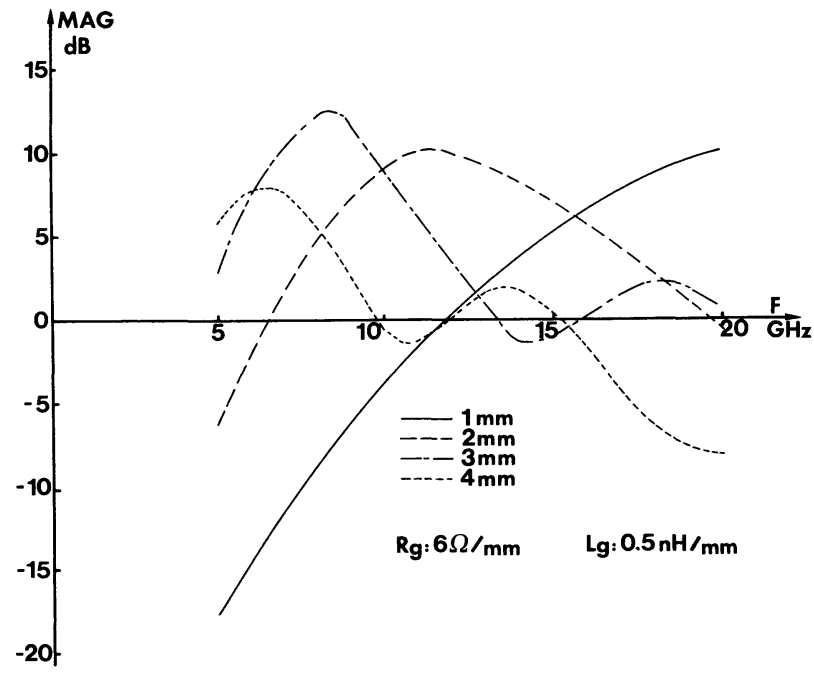

Fig. 8. - Influence du développement de grille $W$.

[Influence of the gate width $W$.]

\subsection{INFLUENCE DE LA POLARISATION DE LA GRILLE} $\mathrm{G}_{2}$. - Dans ce qui précède les polarisations internes des deux monogrilles du schéma intrinsèque équivalent au bigrille étaient supposées identiques, entraînant par conséquent les hypothèses :

$$
g_{\mathrm{m} 1}=g_{\mathrm{m} 2} ; g_{\mathrm{d} 1}=g_{\mathrm{d} 2} ; C_{\mathrm{gs} 1}=C_{\mathrm{gs} 2} ; C_{\mathrm{gd} 1}=C_{\mathrm{gd} 2} .
$$

L'augmentation de la polarisation de la grille $G_{2}$ vers des valeurs plus positives se traduit physiquement par l'ouverture du canal du deuxième monogrille. On a simulé simplement cet effet en augmentant $C_{\mathrm{gs} 2}$ et en diminuant $g_{\mathrm{m} 2}$. A titre d'exemple, la figure 9 montre l'évolution correspondant à $C_{\mathrm{gs} 2}=$ $1,3 C_{\mathrm{gs} 1}$ et $g_{\mathrm{m} 2}=0,25 g_{\mathrm{m} 1}$ pour différents cas de charges $Z_{\mathrm{L} 2}$ et $Z_{\mathrm{L} 4}$. On observe, dans chacun d'eux, un décalage vers les basses fréquences de la réponse 


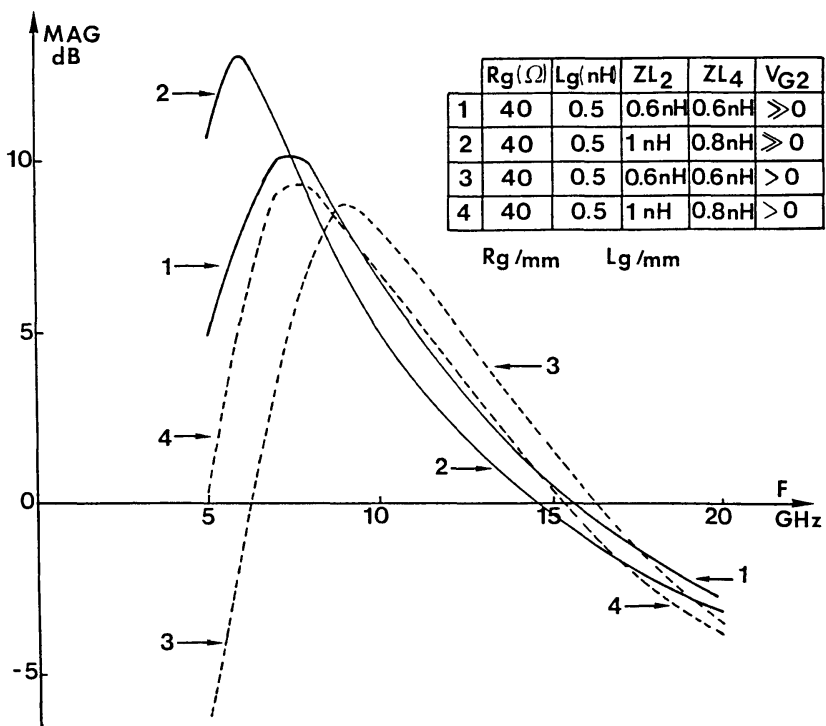

Fig. 9. - Influence de la polarisation de la grille $G_{2}$.

[Influence of the gate $\mathrm{G}_{2}$ biasing.]

du dispositif. Cette propriété est essentielle et constitue un atout important du dispositif qui apparaît ainsi doué d'une accordabilité électronique.

4.5 COMPARAison BIGRILlE-MONOGRILlE. Notre modélisation étant facilement adaptable au traitement du monogrille, nous avons donc pu effectuer une comparaison du comportement de cette structure avec celui de la structure bigrille. Le schéma équivalent utilisé pour représenter chaque cellule élémentaire de la structure monogrille est représenté en encadré sur la figure 10. Pour effectuer la comparaison, on a pris pour self inductances et résistances séries des lignes de grille et de drain du monogrille les mêmes valeurs que pour les deux grilles du bigrille. Les éléments $g_{\mathrm{m}}, C_{\mathrm{gs}}, g_{\mathrm{d}}, C_{\mathrm{gd}}$ et $C_{\mathrm{ds}} \mathrm{du}$ monogrille ont été pris conformément aux égalités :

$$
\begin{aligned}
g_{\mathrm{m}}=g_{\mathrm{m} 1}=g_{\mathrm{m} 2}, & C_{\mathrm{gs}}=C_{\mathrm{gs} 1}=C_{\mathrm{gs} 2}, \\
g_{\mathrm{d}}=g_{\mathrm{d} 1}=g_{\mathrm{d} 2} & C_{\mathrm{gd}}=C_{\mathrm{ds}}=C_{\mathrm{gd} 1}=C_{\mathrm{gd} 2} .
\end{aligned}
$$

La figure 10 montre des exemples typiques de cette comparaison, pour différents couples de valeurs d'impédances de fermeture $Z_{\mathrm{L} 2}$ et $Z_{\mathrm{L} 4}$. De cette comparaison, la conclusion essentielle qui peut être tirée est que la structure bigrille présente un comportement voisin de la structure monogrille avec, toutefois, une bande passante moins étendue et, en particulier, du côté des basses fréquences. Ce résultat n'a rien de surprenant, étant donné que la structure bigrille a un fonctionnement qui se rapproche de celui d'une structure monogrille lorsque la capacité $C_{\mathrm{gs} 2}$ cesse d'avoir un rôle d'isolation entre les lignes d'entrée et de sortie, c'est-à-dire, soit lorsque la fréquence devient élevée, soit lorsque la grille $G_{2}$ est polarisée très positivement.

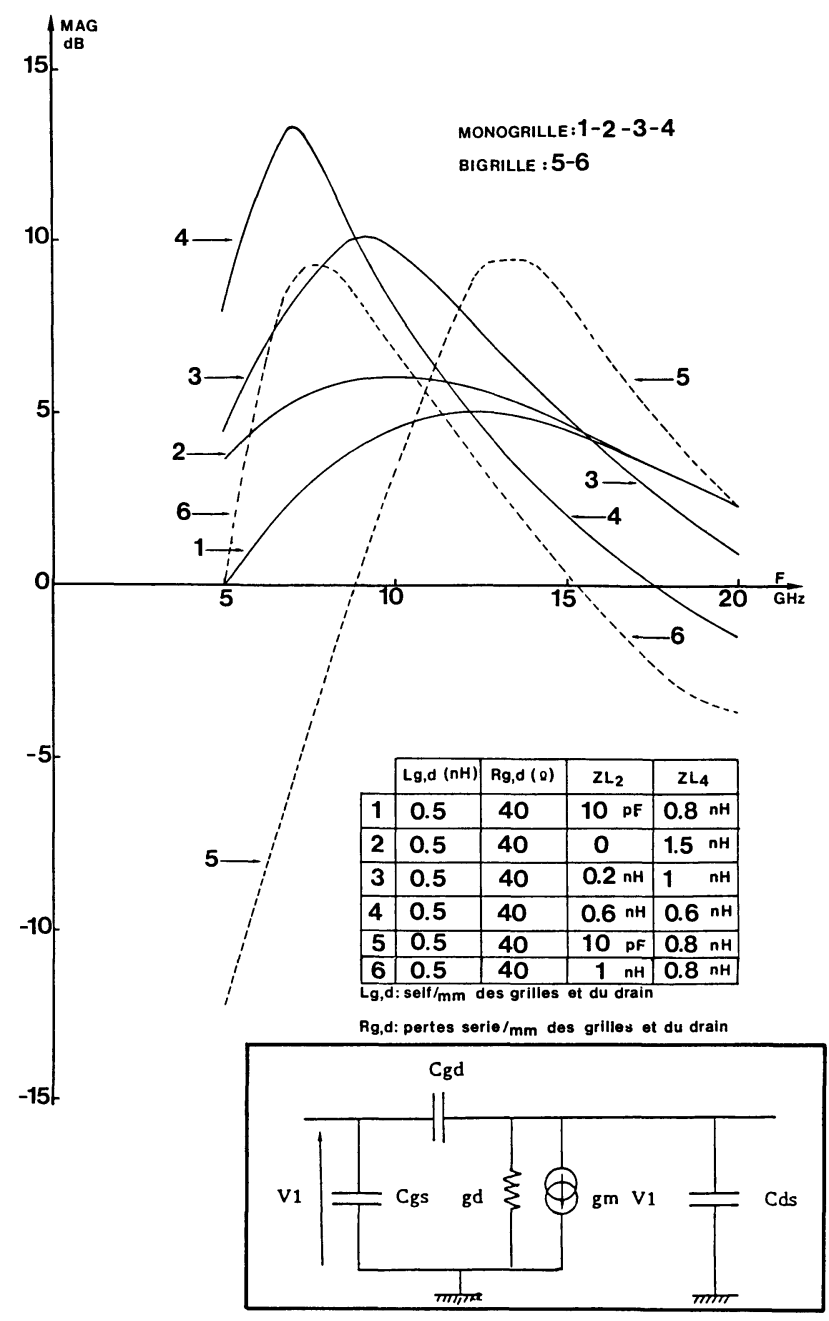

Fig. 10. - Comparaison des structures monogrille et bigrille distribuées.

[Comparison between the single-gate and the dual-gate distributed structures.]

\section{Réalisation et mesures d'une structure d'essai.}

5.1 DESCRIPTION DU DISPOSITIF DE TEST. - Une première réalisation a été effectuée. Il s'agit d'une structure de $1 \mathrm{~mm}$ de développement de grille, avec des longueurs de grille de 2,5 $\mu \mathrm{m}$ dans un intervalle drain-source de $12 \mu \mathrm{m}$. Sa topologie est représentée par la figure 11. Le découplage du drain, sur toute sa

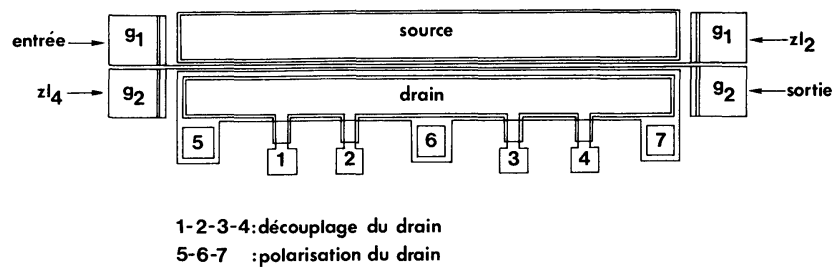

Fig. 11. - Structure bigrille distribuée d'essai.

[Test dual-gate distributed structure.] 
longueur, a été réalisé par une capacité M.I.M. (Métal-Isolant-Métal) de $6000 \AA$ d'épaisseur de polyimide, directement déposée sur la structure. Ses principales caractéristiques électriques sont :

$$
\begin{aligned}
g_{\mathrm{m} 1} & \simeq g_{\mathrm{m} 2} \simeq 60 \mathrm{mS} \\
V_{\mathrm{p}} & =\text { tension } V_{\mathrm{gs} 1} \text { de pincement } \simeq-6 \mathrm{~V} \\
R_{\mathrm{G} 1} & =R_{\mathrm{G} 2}=\text { résistance des grilles } \mathrm{G}_{1} \text { et } \mathrm{G}_{2} \\
& \simeq 20 \text { à } 40 \Omega .
\end{aligned}
$$

Une monture de mesure a été réalisée avec intégration, au niveau de la puce, de la polarisation du drain et des impédances de fermeture $Z_{\mathrm{L} 2}$ et $Z_{\mathrm{L} 4}$. En ce qui concerne ces dernières, nous avons simplement utilisé des fils de connexion pour obtenir des inductances localisées, l'isolation en continu étant réalisée par des chips capacitifs de $50 \mathrm{pF}$ placés en série et ayant une électrode à la masse. Des montures de référence pour les mesures en transmission et en réflexion ont été également réalisées.

5.2 RÉsultats DES MESURES. - La figure 12a donne les résultats obtenus en transmission avec cette structure d'essai. Un comportement passebande, de 3 à $5 \mathrm{GHz}$, est mis en évidence par le comportement d'une part du module du coefficient de transmission $\left(\left|S_{21}\right|\right)$ et, d'autre part, du gain maximum en puissance admissible obtenu expérimentalement en adaptant l'entrée et la sortie de la structure avec des adaptateurs Maury. Un gain

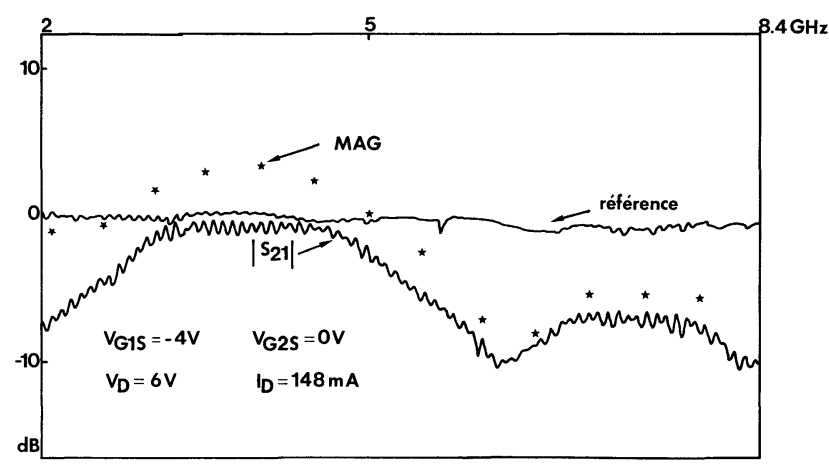

Fig. 12a. - Mesures du coefficient de transmission et du gain maximum en puissance disponible de la structure d'essai.

[Measurements of the transmission coefficient and the maximum available gain of the test structure.]

d'environ $4 \mathrm{~dB}$ a pu être atteint. Les comportements des coefficients de réflexion sont donnés par la figure 12b. Il apparaît que, dans la bande d'amplification de la structure, l'entrée $\left(\left|S_{11}\right|\right)$ est peu désadaptée alors que la sortie $\left(\left|S_{22}\right|\right)$ l'est beaucoup. Le coefficient de transmission inverse $\left(\left|S_{12}\right|\right)$ a été trouvé excellent dans toute l'étendue de la bande de fréquence explorée $(2$ à $8,4 \mathrm{GHz}) \simeq-30 \mathrm{~dB}$.

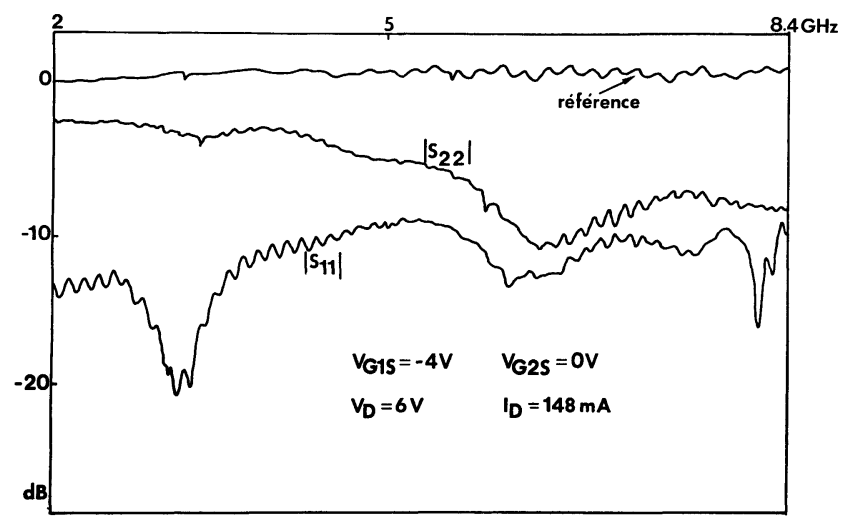

Fig. 12b. - Mesures des coefficients de réflexion d'entrée et de sortie de la structure d'essai.

[Measurements of the input and output reflection coefficients of the test structure.]

5.3 Simulation DE LA STRUCTURE D'ESSAI. Etant donné le caractère relativement basse fréquence de la topologie de la structure d'essai (grilles de $2,5 \mu \mathrm{m}$ de long) une comparaison directe des mesures ne pouvait pas être faite avec les prévisions théoriques développées dans les paragraphes précédents (grilles de $1 \mu \mathrm{m}$ de long). Une simulation a donc été refaite en adaptant les valeurs des paramètres au cas de la structure d'essai. La figure 13 montre les résultats obtenus en prenant :

$$
\begin{array}{rlrl}
g_{\mathrm{m} i} & =70 \mathrm{mS} / \mathrm{mm}, & & C_{\mathrm{gs} i}=2 \mathrm{pF} / \mathrm{mm}, \\
g_{\mathrm{d} i} & =6 \mathrm{mS} / \mathrm{mm}, & & C_{\mathrm{gd} i}=0,2 \mathrm{pF} / \mathrm{mm} \\
L_{\mathrm{g}} & =0,5 \mathrm{nH} / \mathrm{mm}, & R_{\mathrm{g}}=35 \Omega / \mathrm{mm},
\end{array}
$$

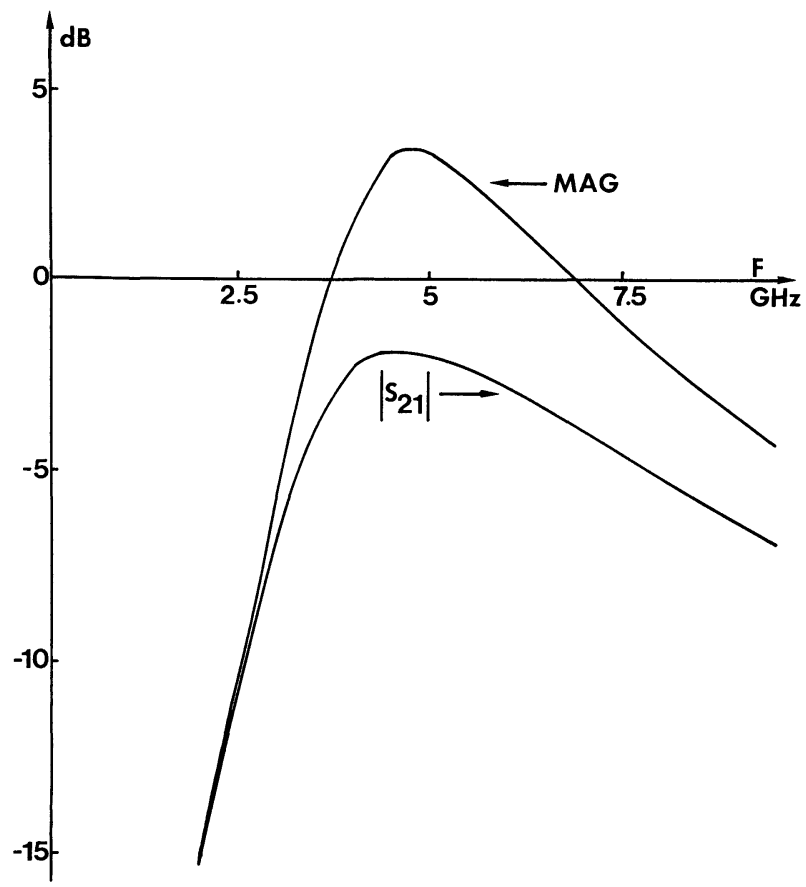

Fig. 13. - Résultats de simulation de la structure d'essai.

[Test structure simulation results.] 
avec des inductances de $1 \mathrm{nH}$ pour les impédances $Z_{\mathrm{L} 2}$ et $Z_{\mathrm{L} 4}$.

Les ordres de grandeurs et les évolutions de $\left|S_{21}\right|$ et de M.A.G. (Maximum Available Gain : gain maximum en puissance disponible) donnés par cette simulation sont globalement en assez bon accord avec les mesures. La validité de la modélisation apparaît donc raisonnablement vérifiée.

\section{Conclusion.}

Sur la base d'une modélisation, de type circuit, permettant de très larges investigations, nous avons montré que la structure bigrille distribuée présente des potentialités d'amplification à large bande. Son comportement est apparu assez voisin de celui de la structure monogrille, son intérêt principal résidant dans la possibilité d'un accord électronique avec la polarisation de la grille $G_{2}$. La réalisation d'une première structure d'essai a permis de tester la validité de l'approche théorique. L'étude doit maintenant se poursuivre avec des réalisations à topologie plus haute fréquence, avec l'objectif d'établir jusqu'où il est possible d'augmenter le développement de grille en produisant une puissance intéressante avec un gain et une bande passante substantiels.

\section{Bibliographie}

[1] Holden, A. J., Daniel, D. R., Davies, I., Oxley, C. H., ReEs, H. D., Gallium Arsenide Travelling Wave Field-Effect Transistors, IEEE ED32, $n^{\circ} 1$ (1985).

[2] Ren, Y. A., Hartnagel, H. L., Travelling wave power FET with coplanar slow-wave electrode system Modeling and experimental results, A.E. U 36 (1982) 468-474.

[3] Heinrich, W., Hartnagel, H. L., Field-theoric analysis of wave propagation on FET electrodes including losses and small-signal amplification, Int. J. Elec. 58, $\mathrm{n}^{\circ} 4$ (1985).

[4] Kretschmer, K. H., Granbow, P., Sigula, T., Coupled-mode analysis of travelling-wave MESFETs, Int. J. Elec. 58, nº 4 (1985).

[5] Baudrand, H., Ahmadpanah, M., Larsry, M., Couplage actif dans une structure à onde lente, J.N.M. (Lannion, France) 1984.

[6] Baudrand, H., Kaddour, M., Ahmadpanah, M., Bias-variable characteristics of coupled coplanar waveguide on $\mathrm{GaAs}$ substrate, $\mathrm{El}$. Lett. $23, \mathrm{n}^{\circ} 4$ (1987).
[7] WYRWINSKI, J., Etude théorique et expérimentale de divers fonctionnements en amplification du transistor à effet de champ bigrille à Arséniure de Gallium, Thèse $3^{\mathrm{e}}$ cycle, Université de Lille I, 1986.

[8] Gamand, P., Crosnier, Y., GÉlin, Ph., Potential Power Performances of the Distributed Amplifiers, 14th European Microwave Conference (Liège) 1984.

[9] Kwon, Y. R., Hietala, V. M., Champlin, K. S., Quasi-TEM analysis of Slow-wave mode propagation on coplanar microstructure MIS Transmission Lines, IEEE MTT35, n 6 (1987).

[10] Krage, M. K., Haddad, G. I., Characteristics of coupled Microstrip transmission lines - II : Evaluation of coupled-line parameters, IEEE MTT 18, $n^{\circ} 4$ (1970).

[11] Fricke, K., HaRtnagel, H. L., Experimental Microwave signal Propagation Study on GaAs MESFET's Using Especially Fabricated Transistors Structures, IEEE EDL-6, n 3 (1985). 